\title{
Status Quo of Piano Coach in China
}

\author{
Yihui Liu
}

University of York, York, UK

\begin{abstract}
The major of piano coach nowadays has a large market demand and is promising in China, and should be attached great importance. However, many music learners in China believe that the students of the piano studies major are sufficiently competent in the work of piano coach, and they often confuse piano coach with piano accompaniment. Although the two majors both require deep understanding of music and good performance skills, they are different in many aspects. The piano coach requires practitioners to have rigorous logic, rich content, corresponding mastery and understanding of the universal law of art and other qualities.
\end{abstract}

Key words: piano art direction; current situation of art direction; domestic piano art direction

The coach major has a huge market demand and is promising in China but in sore need of emphasis by the conservatories of music. Many students studying music now underestimate that coach is an easy task for the piano speciality, and often confuse coach with piano accompaniment. Although the coach major shares music insight and competence of playing with piano performing (piano solo), distinctions still exist. Practitioners should have rigorous logic, abundant experience, and accomplishments in the general rules of art.

\section{Introduction of Piano Coach}

Compared with the conservatories of music in other countries, Chinese colleges of music later established the piano coach major until the 1990s, and now are in a development phase yet. The status quo of this major leads most students a one-sided understanding that coach merely plays a subsidiary role same with the piano accompaniment, and the vocal singer still dominates the performance. Besides, different colleges of music give various appellations to coach as "art accompaniment", "art of piano accompaniment", or "vocal piano accompaniment" etc., which shows their different views on this major. Piano coach and piano performing (piano solo) both are basic constitutions of the piano major, but they are distinguishable in the specialized knowledge. The piano coach can be competent in piano accompaniment, not necessarily vice versa. A qualified piano coach should be adept at piano playing and keyboard and can manage to cope with emergencies in the performance. Meanwhile, various styles and background of the music are all required skills. Therefore, the coach is far more than an auxiliary. Professionally, the coach should be comprehensively qualified with certain knowledge reserve and experience in the music background, styles, playing skills and expression forms. Take Wu Yili, one of the old generation of Chinese musicians, as an example. She is the first generation of pianists in China, also the composer and the first performer of the piano accompaniment of The Butterfly Lovers. Wu Yili has a profound influence on the development of the successors in coach. With the expansion of coach in China in recent years, remarkable improvement can be seen in re-creation in songs by the young practitioners. 


\section{Status Quo of the Piano Coach in China}

2.1 Mis-comprehension of the specialized direction

Most students regard the piano coach major with a superficial understanding that it merely serves to assist the singer in performance. The coach major, in fact, is not only about accompaniment but holds the complementary relationship between the singer and the pianist for the common music aesthetics. Sometimes, the piano coach even leads the role of the associate conductor in music interpretation. As a coach, the practitioner should have a comprehensive quality in music, including the art songs of various countries, chorus, dance, and opera etc. Seen from the vocal performance, the coach is essential in how to treat the performing skills and emotion.

Take Verdi's De miei bollenti spiriti as example, Alfred's love for Violeta in the past few months were demonstrated. In this opera, lots of dotted notes are used, and every note and lyric are a reflection of the character's emotion. Coach, therefore, needs to grasp the overall sentiment and have a deep understanding of the characters.
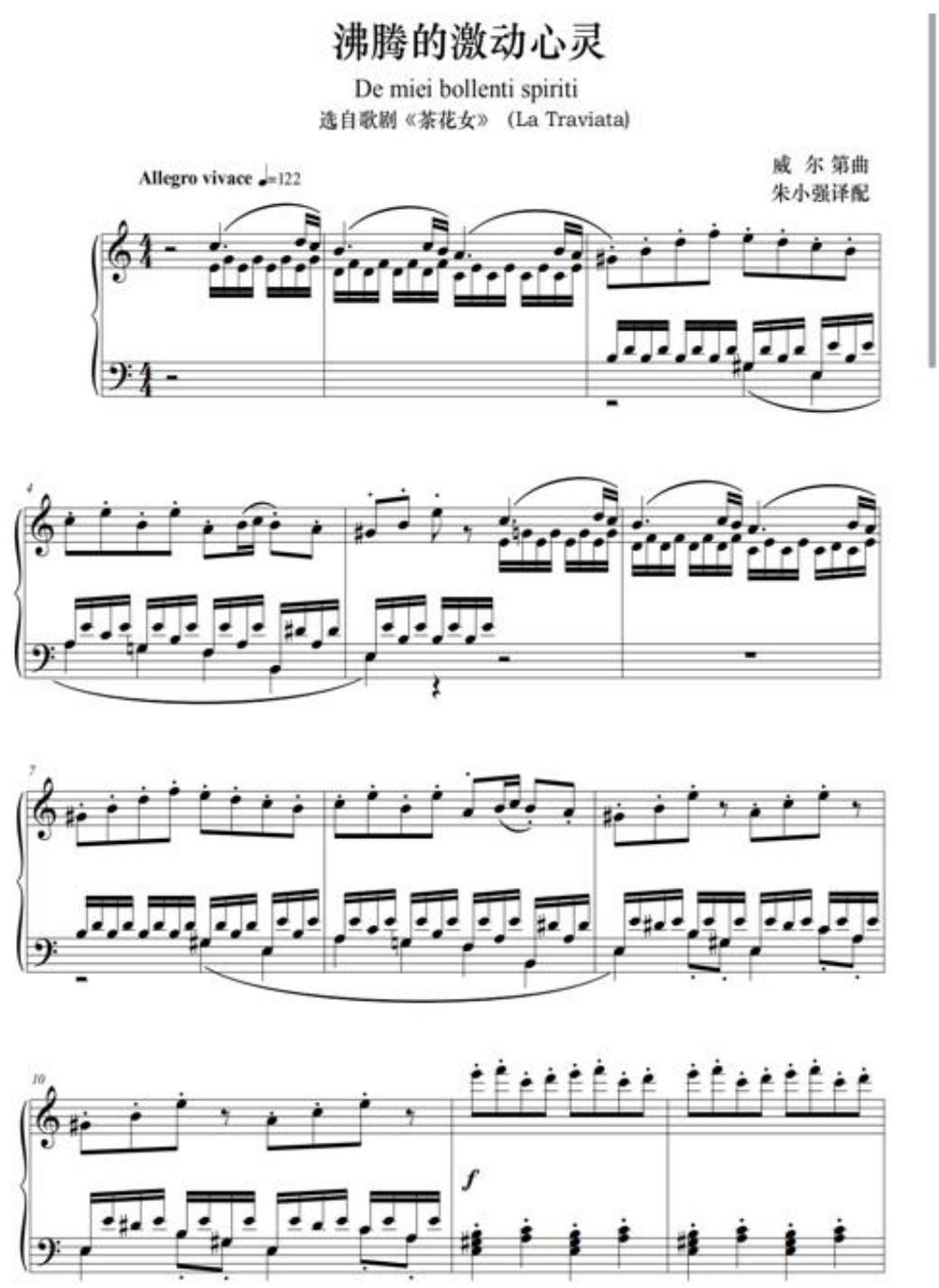

The prelude at the beginning of the song is illustrated by piano simulating the stringed instruments. Smoothly playing with uniform timbre flowing from the left hand and the exact tone colour from the right hand create a quick and enthusiastic atmosphere until the leap part by the right hand. Incremental tension shows Alfred's joy and eagerness. After that, the transition from allegro to medium-fast playing expresses a dramatic effect. 

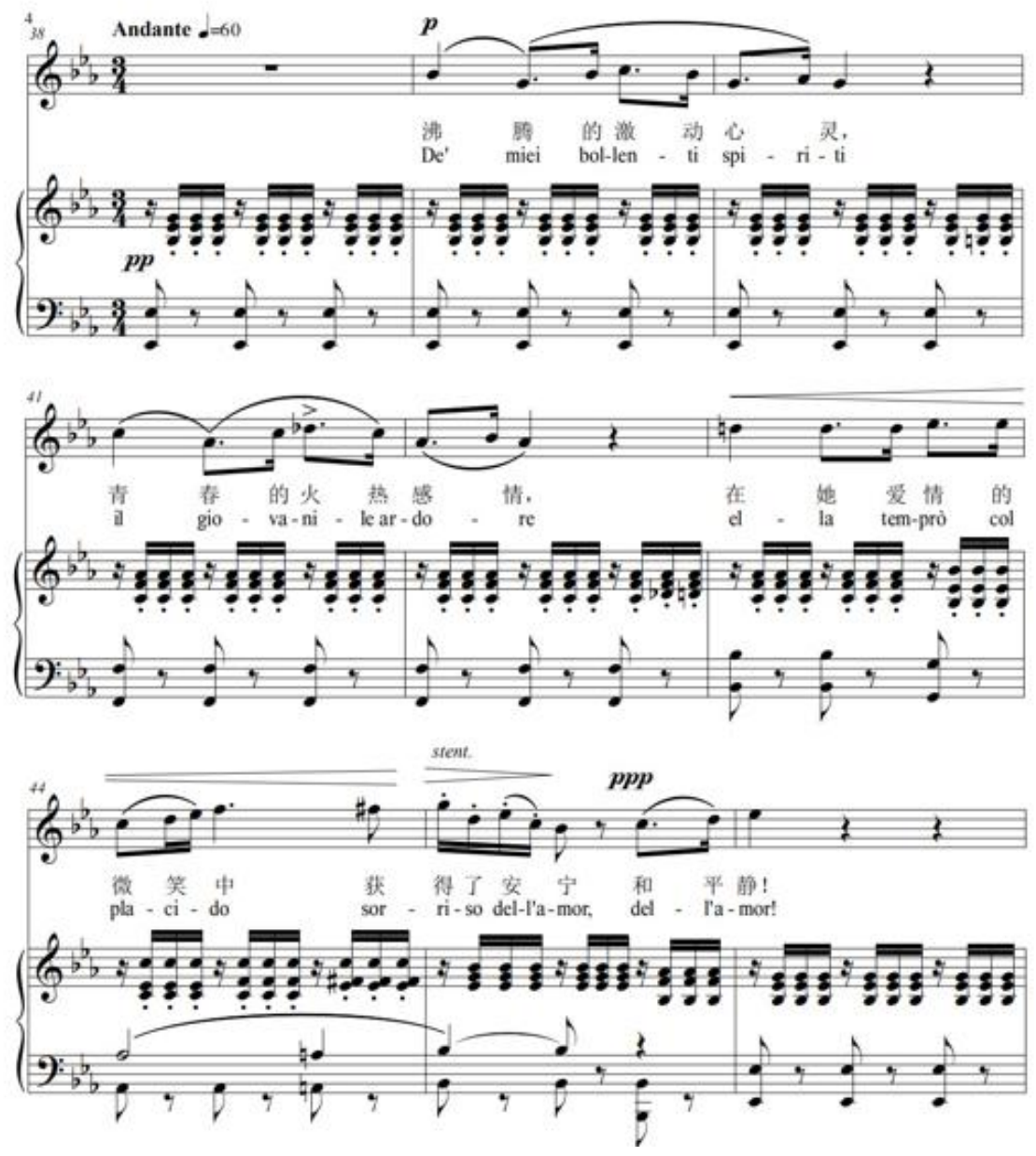

In the aria part, the rhythm ranges from $4 / 4$ to $4 / 3$, which requires a focus of the hands with controlled strength for the coach. The performance of the left hand should be definite and solid full of flexibility. Meanwhile, the coach should be inconsistent with the singer's breath, illustrating Alfred's mood swing through every beat point.

\subsection{Insufficient faculty}

In an early stage of development, few conservatories have set the coach major for specialized talent cultivation, and many vocal music teachers or piano accompanists have to act as the coach, most of whom have no professional training on how to guide singers with better comprehension and illustration in art songs.

\subsection{Insufficiency in comprehensive music accomplishments}

First, a qualified piano coach should be competent in piano performance, keep good control of various figures, terminologies, notes, and the footboard, and manage to cope with emergencies in performance. Besides, the piano coach should have a profound interpretation of the structure, harmony collocation, structural classification of phrase and period of the music work.

Second, the coach should have a robust sight-reading capability, which occupies a large proportion in the enrollment of the coach major by the Chinese conservatories of music. This competence starts with the practice of large numbers of programs of various styles. An intense psychological quality and stress tolerance are also a must. Cooperation with partners is needed for the performance of the coach. Any concerns about the possible errors during the performance may cause nervousness. Therefore, one should learn to calm down and overcome the anxiety with good psychological quality on the stage for better performance. 


\section{Exploration and Findings of the Piano Coach}

Compared with the time-honoured conservatories of music in other countries, there is still much space for improvement of Chinese music for a unique feature. Take the Russian Gnesin's Academy of Music as an example, this century-old academy is founded by the Gnesin family and owns a complete teaching scheme in the coach major. By contrast, the art major in comprehensive universities in China merely lists the coach major as an optional course which is offered in the third year of college. It can be seen that the talent cultivation in coach major hasn't been emphasized by the Chinese universities. By comparison, the Russian Gnesin's Academy of Music sets different courses on the coach for every grade, and students can choose their major courses and preference, which contributes to students' extension in the study with their real love and thirst for music. At the same time, students' music emotion, aesthetic consciousness and individual sentiment get improved.

\section{Conclusions}

This research explored the status quo of the coach in China. Verdi's De miei bollenti spiriti was taken as an example, and the future trend of coach was discussed. Through the parallel comparison with the Russian Gnesin's Academy of Music, it is suggested that the design of major courses in the colleges of music in China can be further improved. We need to learn from the advanced teaching methods of other countries so as to promote the career of the coach.

\section{Conflicts of Interest}

The author declares no conflicts of interest regarding the publication of this paper.

\section{References}

[1] Yuan X.Y. (2019). Significance and Research of the Development of Coach. The Artists, (01):113-114.

[2] Chen M.H. (2019). Characteristics and Cooperation of Art Songs by Professor Zhang Guilin-Academic Forum of "Songs and Piano Accompaniment". Modern Music, (01):126-127.

[3] Wang G.C. (2018). Exploration of the Status Quo of Coach Major in China. Mingrifengshang, (23):139.

[4] Jing Y.Y. (2018). Exploration of Specialized Direction of Coach Major. Appreciation, (29):140-141.

[5] Lei L. (2020). Basic Qualification of Coach. Home Drama, (18):68.

[6] Cheng Y. (2020). The Application of Piano Performance in Vocal Coach-Take Aria Voi, che sapete in Mozart's Le Nozze di Figaro as Example. Appreciation, (09):310-311.

[7] Zhang Z. (2020). Artistic Expression of Aria De miei bollenti spiriti. Home Drama, (22):97-98.

[8] Li X.R. (2020). The Role of Coach in Vocal Performance. Da Guan, (03):17-18.

[9] Lian L.J. (2018). Coach. Dongfang Cangpin (05):76-77.

[10] Fu A. (2013). Comparison Study of the Vocal Coach Course between Russia and China. Northern Music, (06):36-37. 